\title{
The general movements assessment and effects of an early intervention in an infant with Cri du chat syndrome: a case report
}

\author{
Bilge Nur Yardımcı Lokmanoğlu ${ }^{1 \oplus}$, Akmer Mutlu' ${ }^{1 \oplus}$, Ayşe Livanelioğlu' ${ }^{1 \oplus}$, \\ Göknur Haliloğlu ${ }^{20}$ \\ ${ }^{1}$ Developmental and Early Physiotherapy Unit, Hacettepe University, Faculty of Physical Therapy and Rehabilitation; ${ }^{2}$ Department of \\ Pediatric Neurology, Hacettepe University Faculty of Medicine, Ankara, Turkey.
}

\begin{abstract}
Background. Cri du chat syndrome (CdCS) is a rare orphan genetic disorder. Infants with CdCS have a neurodevelopmental dysfunction, but there are limited studies on their spontaneous movements or effect of the early interventions in children with CdCS. This study aimed to describe early spontaneous movements and investigate the effects of an early intervention in an infant with the CdCS.

Case. We analyzed the detailed general movements assessment (GMA) of an infant with CdCS at 14 weeks, and the Bayley Scales of Infant and Toddler Development-third edition (Bayley-III) were used for the determining and the follow-up of developmental functioning at 14 weeks, 6 months and 12 months. The infant was included in an early intervention beginning from 14 weeks. Fidgety movements were absent. The motor repertoire appeared significantly reduced, and the movement character was monotonous at 14 weeks. Although the infant achieved some developmental milestones with the early intervention program, the improvements were not reflected in the Bayley-III composite score.
\end{abstract}

Conclusions. As a consequence, abnormal GMA results, including fidgety movements and concurrent movement patterns, seen in CdCS can be associated with early signs of neurodevelopmental dysfunction. Early intervention programs in infants with genetic disorders could help enable the early achievement of motor milestones.

Key words: cri du chat syndrome, early intervention, general movements, motor development, physiotherapy.

Cri du chat syndrome (CdCS) is a rare orphan genetic disorder occurring as a result of deletions of variable size in the short arm of chromosome 5 (5p-). ${ }^{1}$ The deletion size has been reported to vary between 5 and $40 \mathrm{Mb}$, and severe phenotypic and cognitive impairment is associated with larger deletions. ${ }^{2,3}$ The incidence of CdCS is estimated between 1:15.000 and 1:50.000 live births. ${ }^{4,5}$

The most marked characteristics are facial dysmorphism, high-pitched monotonous

Bilge Nur Yardımcı Lokmanoğlu

bilgenuryardimci@hacettepe.edu.tr

Received 17th April 2020, accepted 20th June 2020. crying, low birth weight, microcephaly, hypotonia, poor growth, developmental delay, delayed motor skill acquisition, clumsiness and intellectual disability. ${ }^{6}$ Asphyxia, hypotonia and feeding difficulties have been reported beginning from the newborn period, and their growth and development are remarkably slow. ${ }^{5}$ As the child gets older, the muscle hypotonia observed in the neonatal period, is replaced with hypertonia, and the microcephaly becomes more prominent., ${ }^{5,6}$ Honjo et al. ${ }^{7}$ reported that neurodevelopmental delay was detected in all of the patients with CdCS. Most commonly associated neuroimaging features include pontine and median cerebellar peduncle hypoplasia, cerebellar vermian atrophy, with additional findings such as; corpus callosum 
dysplasia, mega siterna magna, fourth ventricle enlargement, cerebellar vermian atrophy and delayed myelination. ${ }^{8}$

Of the children with CdCS $72.2 \%$ acquired independent walking at ages ranging from 19 months to 12 years, and in which the same study reported that $20.85 \%$ of the patients, who were older than 2 years old, were not able to walk. ${ }^{7}$ However, it has been reported that these children can achieve many skills despite the delay in various developmental areas. $^{9}$ Although the clinical features and characteristics, and phenotype of CdCS have been reported in several studies, the general movements assessment (GMA) and the effect of an early intervention have not been previously examined. This first case report aimed to; (i) describe the age-specific detailed GMA which includes the fidgety movements and concurrent motor repertoire in a 3 to 5 -month-old infant with CdCS, and (ii) investigate the effect of an early intervention on developmental functioning in an infant with CdCS.

\section{Case Report}

The female infant was born at 40 gestational weeks with a birth weight of $2920 \mathrm{~g}$ by cesarean delivery. She was the first child of a 26-year-old mother. CdCS was suspected from the crying pattern at birth and the family was referred to the Department of Genetics. She stayed in the neonatal intensive care unit for 2 days due to lack of sucking. Karyotype analysis at the age of 3 months revealed CdCS together with 46,XX,del(5)(p14). The patient then was referred to the Department of Gastroenterology for feeding difficulties. She had no hearing or vision problems. The pediatric neurologist referred the patient for physical therapy and rehabilitation when she was 3 months old. She was not able to achieve head control and had hypotonia including reduced antigravity movements, decreased resistance to passive movements, an excessive range of joint mobility in her first evaluation at Developmental and Early Physiotherapy Unit, Faculty of Physical
Therapy and Rehabilitation. Her movements were observed to be quite monotone, slow and repetitive.

The infant was included in an earlyindividualized physiotherapy program that is routinely applied in our unit due to the delay in motor development observed with the initial evaluation (14 weeks). The early intervention was applied for 2 sessions per week for 45 to 60 minutes each time for a total of 9 months in line with the individual motor developmental needs of the infant by an experienced physiotherapist. The objectives of the treatment were identified as postural support, facilitation of movement, developing symmetrical posture, providing muscle balance, transferring weight, and proximal stabilization to support the daily activities of the infant. Positions such as supine, prone and side-lying together with handling techniques and additional sensory stimulation were used in the physiotherapy program in the early period. These were followed by the sitting position, rolling from the supine to the prone position, rolling from the prone to the supine position, and supported standing with the growth of the infant and motor progression. The family was also given a home-program by the physiotherapist on a weekly basis.

When she was 14 weeks old, five-minute video recording was made for the Prechtl GMA which is based on visual gestalt perception and is widely used in the prediction of neurological dysfunction in infants in the earliest period of life. ${ }^{10,11}$ Age-specific detailed GMA which includes fidgety movements and concurrent motor repertoire and postural repertoire were assessed via the video recording. Using the score sheet for the Motor Optimality Score for 3- to 5-Month-Old-Infants-Revised, ${ }^{12}$ we defined a motor optimality score (MOS) with a maximum value of 28 (for the best possible performance) and a minimum value of $5 .^{13}$ The score sheet contains the following five subsections: (i) temporal organization and quality of fidgety movements, (ii) observed movement patterns other than fidgety movements, (iii) ageadequate movement repertoire, (iv) observed 
postural patterns, and (v) movement character. ${ }^{12}$ The video recording was evaluated by two certified raters. Our patient displayed no fidgety movements, and a detailed assessment showed an age-inadequate movement repertoire at this time. Kicking, mouth movement, foot-foot contact were abnormal. The infant showed head and body asymmetry, and abnormal variability of finger postures in the postural patterns. The movement character was monotonous. The MOS was 6/28.

At 14 weeks, 6 months and 12 months old, we applied the Bayley Scales of Infant and Toddler Development-third edition (Bayley-III) that is widely used to measure the developmental functioning of children aged 1 to 42 months. ${ }^{14}$ At 14 weeks the Bayley-III composite score for cognition was 65 (95\% CI: 60-76), for language 79 (95\% CI: 73-88), and 73 (95\% CI: 68-83) for the motor domain. At 6 months and 12 months, the infant achieved several more items in all domains but the age-specific composite scores were still lower than average. At 6 months, the result of her Bayley-III composite score for cognitive domain was 65 (95\% CI: 60-76), for language domain 79 (95\% CI: 73-88), and motor domain 49 (95\% CI: 45-61). According to Bayley-III composite score result at 12 months, cognitive domain was 55 (95\% CI: 51-67), language 62 (95\% CI: 57-72), and 46 (95\% CI: 4358 ) for the motor domain (Table I). Hence, she scored below 2 SD in all domains. The parents gave their informed consent for this publication.

\section{Discussion}

To the best of our knowledge, this is the first case report that describes the fidgety movement and concurrent motor repertoire in an infant with CdCS at the early period and shows the effects of an early intervention on developmental functioning results. Clinical assessments are crucial for predicting later neurodevelopmental outcomes and following functional outcome in long-term development, because neuroimaging techniques such as magnetic resonance imaging (MRI) are not always available for such young infants. Studies showed that brain development continues postnatally, driven by motor cortex activity, ${ }^{15,16}$ therefore, early intervention might play an important role in the improvement in the development of children who have a neurodevelopmental disorder.

Many diseases that are subsumed under the umbrella term of genetic disorders have quite different symptoms, findings or complaints. Several studies in infants with genetic disorders demonstrated that by using the detailed GMA for the assessment of motor repertoire, that these infants might have a wide range MOS result. ${ }^{17-19}$ As in previous studies, MOS was associated with gross motor functional abilities and activity limitations in children with cerebral palsy; ${ }^{20,21}$ fine and gross motor performance in children born with very low birth weight; ${ }^{22}$ cognitive development in children born preterm; ${ }^{23}$ and, with language performance in typically developing children, ${ }^{24}$ hence these differences in MOS results in infants with genetic disorders might be an early indicator of their later neurodevelopmental level. According to our findings, GMA results were abnormal at 14 weeks, which revealed also significantly reduced MOS like the other genetic disorders such as Smith Magenis syndrome, ${ }^{17}$ some infants with Down syndrome ${ }^{18}$ and Prader Willi Syndrome. ${ }^{19}$ Lacking variable and complex quality of general movements indicate an abnormality in the nervous system. ${ }^{25}$ Underlying mechanisms of fidgety movements and the resulting low MOS are unclear in infants with CdCS, but our case report is a contribution to the literature.

Mainardi et al. ${ }^{9}$ reported that all their patients with CdCS had motor delay, and the severity of motor delay was related to the size of the deletion. Furthermore, $81.9 \%$ of the patients were diagnosed in the first year of life, and it was reported that $71.6 \%$ of the patients started physiotherapy before one year-old in the same study. ${ }^{9}$ Although patients who started physiotherapy before one year-old are indicated to have better results in the achievement of developmental skills, there was no statistical 


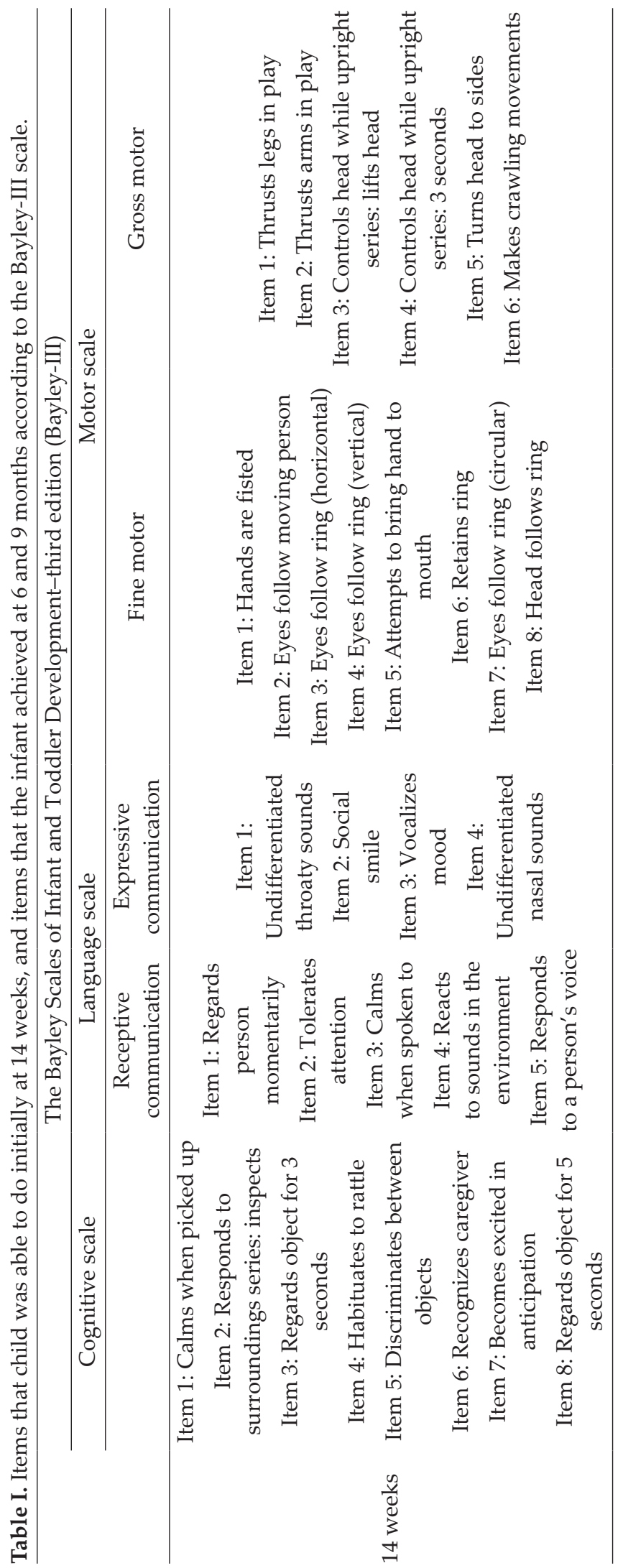




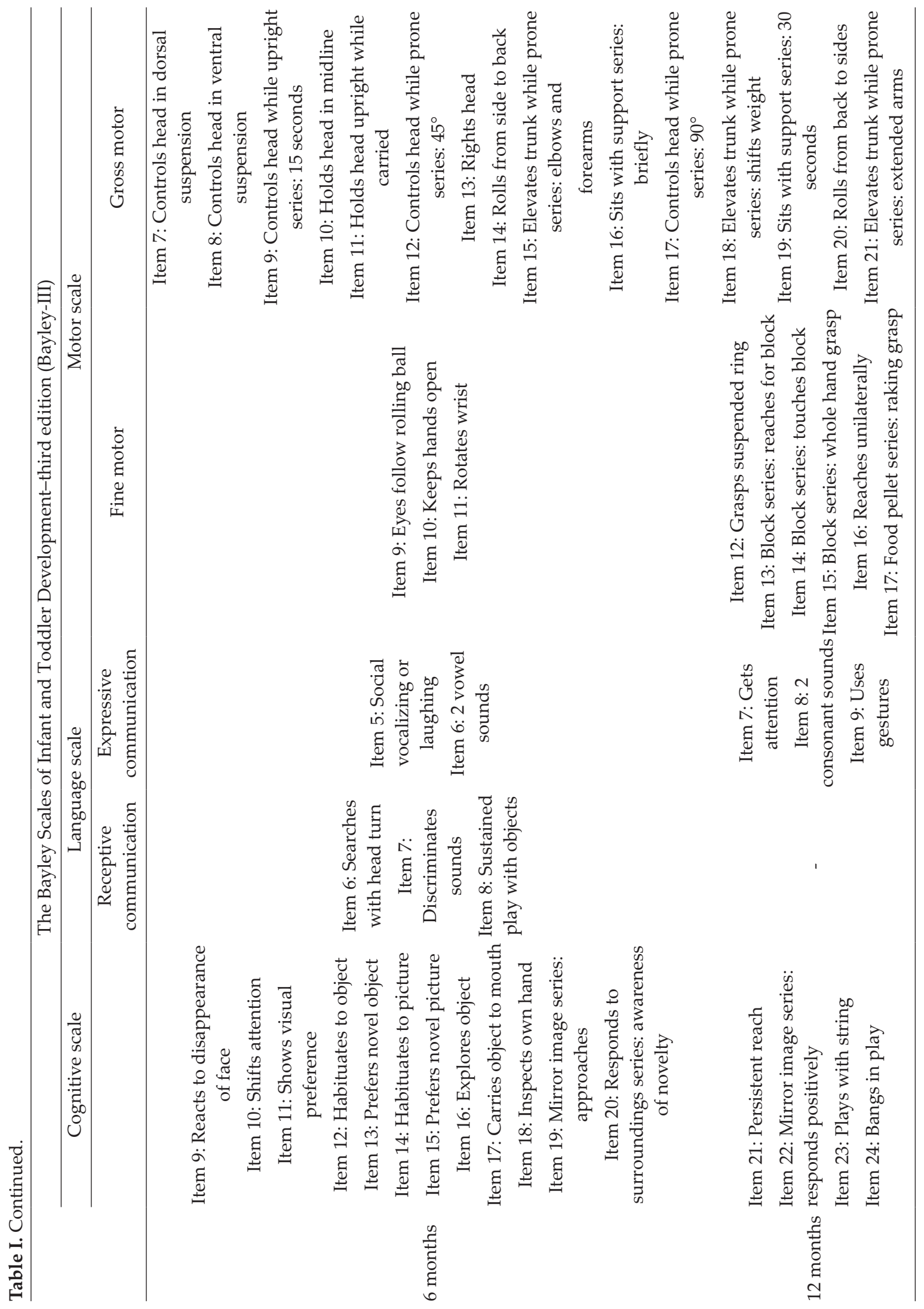


significance. ${ }^{9}$ Guala et al. ${ }^{26}$ who carried out another study, investigated the effect of two rehabilitation programs on the development and reported that no significant difference was found. There are several findings concerning physiotherapy, ${ }^{9,26}$ but some information in these studies is unclear, such as specific and detailed information about the physiotherapy, the child's initial functional levels, and age of onset of physiotherapy. Our findings contribute to the literature that early-individualized physiotherapy programs might be effective in achieving developmental milestones.

Furthermore, the improvement of our infant's development, especially in the motor and cognitive domains, in the first months of life was also more than the subsequent periods with physiotherapy, for this reason, we point out that starting rehabilitation in the first 6 months is very important. According to our findings, although our infant achieved some developmental milestones, these achievements were not reflected in the Bayley-III composite score results. The improvements in the BayleyIII raw score results were not enough to catch up with her peers.

We recognize several limitations to this case report. Firstly, we did not obtain a cranial magnetic resonance imaging study, because of the age of the infant and the need for anesthesia. Secondly, our findings cannot be generalized because they are based on only one infant.

In conclusion, apart from the clinical features and characteristics and phenotype of CdCS, our findings demonstrated that abnormal GMA results, including fidgety movements and concurrent movement and postural patterns, might be associated with malformations of the central nervous system development at the earliest age in infants with CdCS. Additionally, according to our findings, early-individualized intervention might be important to reduce the neurodevelopmental delays of these children and to achieve the developmental milestones earlier. Whether these improvements in our infant are effects of the early intervention or just a result of the normal growth and development process is controversial as in the whole pediatric group. Our findings need to be verified in a large cohort.

\section{REFERENCES}

1. Cerruti Mainardi P. Cri du Chat syndrome. Orphanet J Rare Dis 2006; 1: 33.

2. Simmons AD, Püschel AW, McPherson JD, Overhauser J, Lovett M. Molecular cloning and mapping of human semaphorin $\mathrm{F}$ from the Cridu-chat candidate interval. Biochem Biophys Res Commun 1998; 242: 685-691.

3. Wilkins LE, Brown JA, Nance WE, Wolf B. Clinical heterogeneity in 80 home-reared children with cri du chat syndrome. J Pediatr 1983; 102: 528-533.

4. Higurashi M, Oda M, Iijima $K$, et al. Livebirth prevalence and follow-up of malformation syndromes in 27,472 newborns. Brain Dev 1990; 12: 770-773.

5. Niebuhr E. The Cri du Chat syndrome: epidemiology, cytogenetics, and clinical features. Hum Genet 1978; 44: 227-275.

6. Mainardi PC, Perfumo C, Calì A, et al. Clinical and molecular characterisation of 80 patients with $5 p$ deletion: genotype-phenotype correlation. J Med Genet 2001; 38: 151-158.

7. Honjo RS, Mello CB, Pimenta LSE, et al. Cri du Chat syndrome: characteristics of 73 Brazilian patients. J Intellect Disabil Res 2018; 62: 467-473.

8. Correa DG, Ventura N, Gasparetto EL. Pontine hypoplasia in cri-du-chat syndrome: alterations in diffusion tensor imaging. Childs Nerv Syst 2017; 33: 1241-1242.

9. Mainardi PC, Pastore G, Castronovo C, et al. The natural history of cri du chat syndrome. A report from the Italian Register. Eur J Med Genet 2006; 49: 363-383.

10. Prechtl HF, Einspieler C, Cioni G, Bos AF, Ferrari F, Sontheimer D. An early marker for neurological deficits after perinatal brain lesions. Lancet 1997; 349: 1361-1363.

11. Novak I, Morgan C, Adde L, et al. Early, accurate diagnosis and early intervention in cerebral palsy: advances in diagnosis and treatment. JAMA Pediatr 2017; 171: 897-907. 
12. Einspieler C, Bos AF, Krieber-Tomantschger M, et al. Cerebral palsy: early markers of clinical phenotype and functional outcome. J Clin Med 2019; 8: 1616.

13. Einspieler C, Prechtl HFR, Bos AF, Ferrari F, Cioni G. Prechtl's Method on the Qualitative Assessment of General Movements in Preterm, Term and Young Infants. (Clinics in Developmental Medicine No. 167), London: Mac Keith Press, 2004

14. Bayley N. Bayley Scales of Infant and Toddler Development (Bayley-III). (3rd ed). San Antonia, TX, USA: PsychCorp, Pearson, Harcourt Assessment, 2006.

15. Martin JH, Chakrabarty S, Friel KM. Harnessing activity-dependent plasticity to repair the damaged corticospinal tract in an animal model of cerebral palsy. Dev Med Child Neurol 2011;53(Suppl 4): 9-13.

16. Eyre J. Corticospinal tract development and activity dependent plasticity. In: Shepherd R (ed). Cerebral Palsy in Infancy. Churchill Livingstone, Elsevier Health Sciences, 2014: 51-70.

17. Einspieler C, Hirota H, Yuge M, Dejima S, Marschik PB. Early behavioural manifestation of SmithMagenis syndrome (del 17p11. 2) in a 4-month-old boy. Dev Neurorehabil 2012; 15: 313-316.

18. Herrero D, Einspieler C, Panvequio Aizawa CY, et al; GenGM Study Group. The motor repertoire in 3-to 5-month old infants with Down syndrome. Res Dev Disabil 2017; 67: 1-8.

19. Pansy J, Barones C, Urlesberger B, et al. Early motor and pre-linguistic verbal development in PraderWilli syndrome - A case report. Res Dev Disabil 2019; 88: 16-21.
20. Bruggink JL, Cioni G, Einspieler C, Maathuis CG, Pascale R, Bos AF. Early motor repertoire is related to level of self-mobility in children with cerebral palsy at school age. Dev Med Child Neurol 2009; 51: 878-885.

21. Yang H, Einspieler C, Shi W, et al. Cerebral palsy in children: movements and postures during early infancy dependent on preterm vs. full term births. Early Hum Dev 2012; 88: 837-843.

22. Zang FF, Yang H, Han Q, et al. Very low birth weight infants in China: the predictive value of the motor repertoire at 3 to 5 months for the motor performance at 12 months. Early Hum Dev 2016; 100: 27-32.

23. Butcher PR, van Braekel K, Bouma A, Einspieler C, Stremmelaar EF, Bos AF. The quality of preterm infants' spontaneous movements: an early indicator of intelligence and behaviour at school age. J Child Psychol Psychiatry 2009; 50: 920-930.

24. Salavati S, Einspieler C, Vagelli G, et al. The association between the early motor repertoire and language development in term children born after normal pregnancy. Early Hum Dev 2017; 111: 30-35.

25. Peyton C, Einspieler C. General movements: a behavioral biomarker of later motor and cognitive dysfunction in NICU graduates. Pediatr Ann 2018; 47: e159-e164.

26. Guala A, Spunton M, Tognon F, et al. Psychomotor development in cri du chat syndrome: comparison in two Italian cohorts with different rehabilitation methods. Sci World J 2016; 2016: 3125283. 